\title{
Time-Frequency Root-MUSIC Method Based on Pseudo Wigner-Ville Distribution for Near-Field Source Localization
}

\author{
Xin Sun ${ }^{1}$, Hong Jiang ${ }^{2}$, Bo Wang ${ }^{3}$ \\ College of Communication Engineering, Jilin University, Changchun, China \\ ${ }^{1}$ sunxin0529@163.com, ${ }^{*}$ jiangh@jlu.edu.cn, ${ }^{3}$ roywang@jlu.edu.cn
}

\begin{abstract}
In this paper, a novel time-frequency Root-MUSIC method for near-field source localization is proposed. In the method, two spatial time-frequency matrices of Pseudo Wigner-Ville distribution (PWVD) are constructed, and a time-frequency RootMUSIC algorithm is developed to estimate bearings and ranges of near-field targets. It has high accuracy and resolution for the bearing and range estimation of non-stationary signals, even when the signalto-noise ratio (SNR) is low, or the sources are closely spaced. The effectivity of the method is validated by simulations.

Index Terms - near-field source localization; time-frequecy distribution; parameter estimation; Root-MUSIC.
\end{abstract}

\section{Introduction}

Spatial source parameter estimation has been an important research field of array signal processing over the decades. It is widely used in radar, sonar, communications and medical imaging. Most of the array processing methods assume that the sources are in the far-field and the arrays are in the same plane. However, when the sources are in the nearfield, the assumption of plane wave no longer holds.

The research on near-field source localization is originated in the late 70 s, early 80 s of last century. The estimation methods mainly include triangle methods and time delay estimation methods. Delay estimation methods include beamforming method $^{[1]}$, maximum entropy method, maximum likelihood method, Capon method ${ }^{[2]}$, linear prediction class method, MUSIC method, eigenvector method, path following method $^{[3]}$, ESPRIT method, higher order cumulant method ${ }^{[4]}$, circular correlation method ${ }^{[5]}$, far-field estimation methods, and so on.

Time-frequency analysis has been considered by scholars as a very effective technology for non-stationary signals (e.g.linear frequency modulation (LFM)) processing in the last decade $^{[6-8]}$. G. M. Amin, Yimin. Zhang and others have done a lot of research on time-frequency analysis for blind source separation and DOA estimation ${ }^{[9-11]}$, but very few for the nearfield application ${ }^{[12-14]}$.

In this paper, we propose a near-field time-frequency Root-MUSIC method, aiming to improve the performance of estimation of bearing and range of non-stationary signal in the near-field. Two spatial time-frequency matrices of Pseudo Wigner-Ville distribution (PWVD) are constructed, and a time-frequency Root-MUSIC algorithm is developed to

*Corresponding author: Hong Jiang, email: jiangh@jlu.edu.cn estimate bearings and ranges of near-field targets. The method is validated by simulation.

\section{Wigner-Ville distribution of the signals}

A linear frequency modulation (LFM) signal is shown in Eq.(1)

$$
S(t)=e^{j 2 \pi\left(f_{0} t+\frac{1}{2} k t^{2}\right)}
$$

where $f_{0}$ and $k$ are starting frequency and FM rate of $s(t)$.

The Wigner-Ville distribution (WVD) of $s(t)$ is

$$
\begin{aligned}
W(t, f)= & \int_{-\infty}^{+\infty} s\left(t+\frac{\tau}{2}\right) s^{*}\left(t-\frac{\tau}{2}\right) e^{-j 2 \pi f \tau} d \tau \\
& =\int_{-\infty}^{+\infty} e^{j 2 \pi\left(f_{0}+k t\right) \tau} e^{-j 2 \pi f \tau} d \tau \\
& =\delta\left(f-\left(f_{0}+k t\right)\right)
\end{aligned}
$$

Therefore, in the time-frequency domain, the WVD of a LFM signal is a straight line changed by the FM rate. The WVD spectrum of a LFM signal is given by Fig.1.

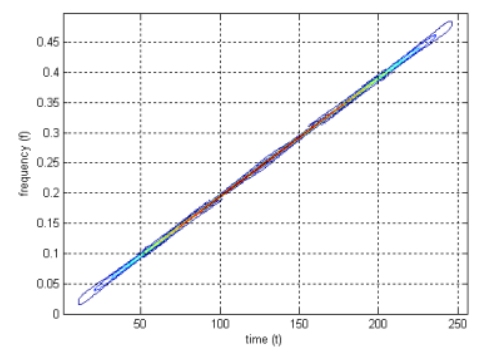

(a) contour plot

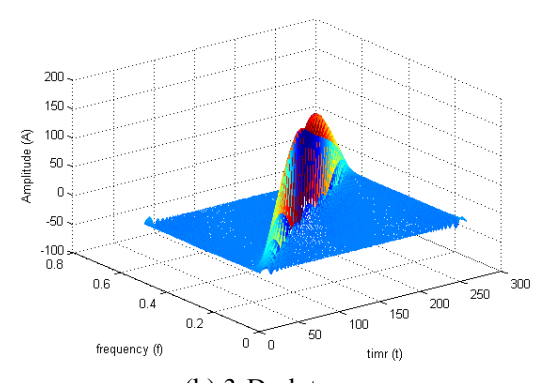

(b) 3-D plot

Fig.1 The WVD spectrum of a LFM signal 


\section{Array Model}

The following assumptions are assumed to hold:

1) The source signals are LFM signals, and are independent with each other. The positioning parameters of different sources are different, and the received signals are the superposition of a series of spherical wave.

2) The noise received by each array element is stationary additive white Gaussian noise, its mean is zero and variance is $\sigma^{2}$. The noises between different array elements are independent, so are with the signal sources.

3) The spacing of array elements $d$ is not greater than the $1 / 4$ of the wavelength $\lambda$, that is, $d \leq \lambda / 4$, and the number of signal sources $\mathrm{P}$ is less than half the number of array elements $\mathrm{M}$, that is, $P<M / 2$.

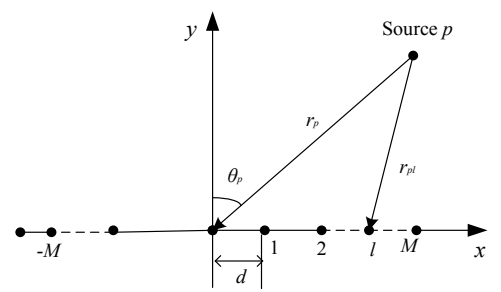

Fig. 2. The model of near-field source localization

As shown in Fig.2, we consider a sensor array consisting of $L=2 M$ sensors, the spacing of sensors is $d$. The sensors receive $P$ narrowband and irrelevant signal sources. We choose the sensor with number 0 as a phase reference point. The bearing and range of $p$-th signal source is $\left(\theta_{p}, r_{p}\right)$. In the presence of additive noise, the signal received by the $l$-th sensor can be expressed as

$$
\begin{aligned}
x_{l}(t)= & \sum_{p=1}^{P} b_{p} s_{p}(t) e^{j \tau_{p l}}+n_{l}(t) \\
& =\sum_{p=1}^{P} b_{p} s_{p}(t) e^{j \omega_{p} l+j \phi_{p} l^{2}}+n_{l}(t)
\end{aligned}
$$

for $-M+1 \leq l \leq M$, where, $s_{p}(t)$ is the $p$-th signal source, $b_{p}$ is the amplitude of the $p$-th signal. $n_{l}(t)$ is an additive white Gaussian noise. $\tau_{p l}=\omega_{p} l+\phi_{p} l^{2}$ is the propagation delay of the $p$-th signal source between the $l$-th array element and the reference array element.

$$
\begin{aligned}
& \omega_{p}=-2 \pi \frac{d}{\lambda} \sin \theta_{p} \\
& \phi_{p}=\pi \frac{d^{2}}{\lambda r_{p}} \cos ^{2}\left(\theta_{p}\right)
\end{aligned}
$$

where $\lambda$ is the wavelength. $\omega_{p}$ is the nonlinear function of $\theta_{p}$, and $\phi_{p}$ is the nonlinear function of both $\theta_{p}$ and $r_{p}$.

\section{Near-Field Time-Frequency Root-Music Method}

A time-frequency distribution matrix by Pseudo WignerVille distributions (PWVD) with a rectangular window of length is given by [11]. Based on it, we express the time- frequency distribution of $x_{l}(t)$ as

$$
D_{x_{l}, x_{l}}(t, f)=\sum_{\tau=-(h-1) / 2}^{(h-1) / 2} x_{l}(t+\tau) x_{l}^{*}(t-\tau) e^{-j 4 \pi f \tau}
$$

Then we construct two matrices $\mathbf{D}_{1}$ and $\mathbf{D}_{2}$ using spatial time-frequency distribution matrices, where the $\left(l_{1}, l_{2}\right)$-th elements of the two matrices respectively are

$$
\begin{aligned}
& \mathbf{D}_{1}\left(l_{1}, l_{2}\right)=E\left[D_{x_{1-1}, x_{x_{2-1}}}(t, f)\right] \\
& =E\left[\sum_{\tau=(h-1) / 2}^{(h-1) / 2} x_{l_{1}-l_{2}}(t+\tau) x_{L_{-}-l_{1}}^{*}(t-\tau) e^{-j 4 \pi f \tau}\right]
\end{aligned}
$$

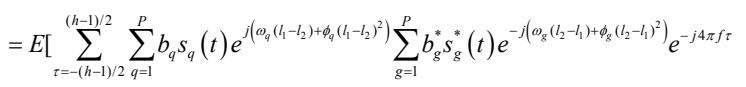

$$
\begin{aligned}
& \left.+\sum_{\tau=-(h-1) / 2}^{(h-1) / 2} n_{l_{1-1}}(t+\tau) n_{l_{2}-1}^{*}(t-\tau) e^{-j 4 \pi f \tau}\right] \\
& =\sum_{p=1}^{P} E\left[D_{s_{p}}(t, f)\right] e^{2 j \omega_{p}\left(l_{1}-l_{2}\right)}+E\left[D_{n}(t, f)\right] \\
& =\sum_{p=1}^{P} E\left[D_{s_{p}}(t, f)\right] e^{2 j \omega_{p}\left(l_{1}-l_{2}\right)}+\sigma^{2} \delta_{l_{1}, l_{2}} \\
& \mathbf{D}_{2}\left(l_{1}, l_{2}\right)=E\left[D_{x_{1-2-1+1}, x_{1-1-2}}(t, f)\right] \\
& =E\left[\sum_{\tau=(h-1) / 2}^{(h-1) / 2} x_{1-1-l_{2}+1}(t+\tau) x_{l_{1}-l_{2}}^{*}(t-\tau) e^{-j 4 \pi f \tau}\right]
\end{aligned}
$$

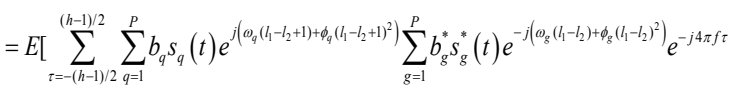

$$
\begin{aligned}
& \left.+\sum_{t=-(h-1) / 2}^{(h-1) / 2} n_{l_{1}-L_{2}+1}(t+\tau) n_{n_{1}-l_{2}}^{*}(t-\tau) e^{-j 4 \pi f \tau}\right] \\
& =\sum_{p=1}^{P} E\left[D_{s_{p}}(t, f)\right] e^{j \theta_{p}} e^{j \phi_{p}} e^{2 j \phi_{p}\left(1-L_{2}\right)}+E\left[D_{n}(t, f)\right] \\
& =\sum_{p=1}^{P} E\left[D_{s_{p}}(t, f)\right] e^{j \omega_{p}} e^{j \phi_{p}} e^{2 j \phi_{p}\left(l_{1}-L_{2}\right)}+\sigma^{2} \delta_{l, l_{2}}
\end{aligned}
$$

where $l_{1}=1, \cdots, M, \quad l_{2}=1, \cdots, M$. Thus, the two matrixes $\mathbf{D}_{1}$ and $\mathbf{D}_{2}$ can be further written as

$$
\begin{aligned}
& \mathbf{D}_{1}=\mathbf{A}(\omega) E\left[\mathbf{D}_{s}(t, f)\right] \mathbf{A}^{H}(\omega)+\sigma^{2} \mathbf{I} \\
& \mathbf{D}_{2}=\mathbf{A}(\phi) E\left[\mathbf{D}_{s}(t, f)\right] \Omega \Phi \mathbf{A}^{H}(\phi)+\sigma^{2} \mathbf{I}
\end{aligned}
$$

where

$$
\begin{aligned}
\mathbf{D}_{s}(t, f) & =\operatorname{diag}\left(\mathbf{D}_{s_{1}}(t, f), \cdots, \mathbf{D}_{s_{P}}(t, f)\right) \\
\mathbf{A}(\omega) & =\left[\mathbf{a}\left(\omega_{1}\right), \mathbf{a}\left(\omega_{2}\right), \cdots, \mathbf{a}\left(\omega_{P}\right)\right] \\
\mathbf{A}(\phi) & =\left[\mathbf{a}\left(\phi_{1}\right), \mathbf{a}\left(\phi_{2}\right), \cdots, \mathbf{a}\left(\phi_{P}\right)\right] \\
\mathbf{a}\left(\omega_{p}\right) & =\left[1, e^{2 j \omega_{p}}, \cdots, e^{2 j(M-1) \omega_{p}}\right]^{T} \\
\mathbf{a}\left(\phi_{p}\right) & =\left[1, e^{2 j \phi_{p}}, \cdots, e^{2 j(M-1) \phi_{p}}\right]^{T} \\
\Omega & =\operatorname{diag}\left(e^{j \omega_{1}}, \cdots, e^{j \omega_{P}}\right) \\
\Phi & =\operatorname{diag}\left(e^{j \phi_{1}}, \cdots, e^{j \phi_{P}}\right)
\end{aligned}
$$

Perform the eigenvalue decomposition of $\mathbf{D}_{1}$ and $\mathbf{D}_{2}$,

$$
\begin{aligned}
& \mathbf{D}_{1}=\left[\begin{array}{ll}
\mathbf{E}_{S 1} & \mathbf{E}_{N 1}
\end{array}\right] \mathbf{\Lambda}_{1}\left[\begin{array}{ll}
\mathbf{E}_{S 1} & \mathbf{E}_{N 1}
\end{array}\right]^{H} \\
& \mathbf{D}_{2}=\left[\begin{array}{ll}
\mathbf{E}_{S 2} & \mathbf{E}_{N 2}
\end{array}\right] \boldsymbol{\Lambda}_{2}\left[\begin{array}{ll}
\mathbf{E}_{S 2} & \mathbf{E}_{N 2}
\end{array}\right]^{H}
\end{aligned}
$$

where, $\boldsymbol{\Lambda}_{1}$ and $\boldsymbol{\Lambda}_{2}$ are the eigenvalues of the matrices $\mathbf{D}_{1}$ and $\mathbf{D}_{2}$, respectively. $\mathbf{E}_{S 1}$ and $\mathbf{E}_{s 2}$ are signal subspaces consisting of the largest $P$ eigenvalues of $\mathbf{D}_{1}$ and $\mathbf{D}_{2}$, and $\mathbf{E}_{N 1}$ 
and $\mathbf{E}_{N 2}$ are noise subspaces consisting by the minimum $M-P$ eigenvalues, respectively. $\mathbf{E}_{S 1}, \mathbf{E}_{S 2} \in \mathbf{C}^{M \times P}, \mathbf{E}_{N 1}, \mathbf{E}_{N 2} \in \mathbf{C}^{M \times(M-P)}$.

Eq.(19) can be rewritten as

From Eq.(10),

$$
\mathbf{D}_{2}=\mathbf{E}_{S 2} \Lambda_{2} \mathbf{E}_{S 2}^{H}+\mathbf{E}_{N 2} \Lambda_{2} \mathbf{E}_{N 2}^{H}
$$

$$
\mathbf{D}_{2} \mathbf{E}_{N 2}=\mathbf{A}(\phi) E\left[\mathbf{D}_{s}(t, f)\right] \Omega \Phi \mathbf{A}^{H}(\phi) \mathbf{E}_{N 2}+\sigma^{2} \mathbf{E}_{N 2}
$$

From Eq.(20),

$$
\mathbf{D}_{2} \mathbf{E}_{N 2}=\mathbf{E}_{S 2} \Lambda_{2} \mathbf{E}_{S 2}^{H} \mathbf{E}_{N 2}+\mathbf{E}_{N 2} \Lambda_{2}=0+\mathbf{E}_{N 2} \Lambda_{2}=\sigma^{2} \mathbf{E}_{N 2}
$$

From Eqs.(21) and (22),

$$
\mathbf{A}(\phi) E\left[\mathbf{D}_{s}(t, f)\right] \Omega \Phi \mathbf{A}^{H}(\phi) \mathbf{E}_{N 2}=0
$$

Since $E\left[\mathbf{D}_{s}(t, f)\right], \Omega$ and $\Phi$ are all full rank matrices, Eq.(23) becomes into

$$
\mathbf{A}^{H}(\phi) \mathbf{E}_{N 2}=0
$$

That is

$$
\mathbf{E}_{N 2}^{H} \mathbf{a}\left(\phi_{p}\right)=0
$$

It can be seen that the noise subspace and signal steering vector are orthogonal.

Refer to a near-field Root-MUSIC method proposed in [15], let $\alpha=e^{2 j \omega}, \beta=e^{2 j \phi}$, and $\delta=\mathbf{E}_{N 1} \mathbf{E}_{N 1}{ }^{H}, \quad \gamma=\mathbf{E}_{N 2} \mathbf{E}_{N 2}{ }^{H}$, we obtain two polynomials as

$$
\begin{aligned}
\mathbf{F}(\alpha)= & {\left[\begin{array}{llll}
1 & \alpha & \cdots & \alpha^{M-1}
\end{array}\right]^{*} \delta\left[\begin{array}{llll}
1 & \alpha & \cdots & \alpha^{M-1}
\end{array}\right]^{T} } \\
& =\sum_{K=0}^{M-1} \delta_{K}^{\prime} \alpha^{K} \\
\mathbf{F}(\beta)= & {\left[\begin{array}{llllll}
1 & \beta & \cdots & \beta^{M-1}
\end{array}\right]^{*} \gamma\left[\begin{array}{lllll}
1 & \beta & \cdots & \beta^{M-1}
\end{array}\right]^{T} } \\
& =\sum_{K=0}^{M-1} \gamma_{K}^{\prime} \beta^{K}
\end{aligned}
$$

where, the coefficients $\delta_{K}^{\prime}$ and $\gamma_{K}^{\prime}$ are the sum of the elements along the diagonals of $\delta$ and $\gamma$, respectively. Using Eq.(26) and Eq.(27) to extract their roots, we can achieve $\alpha=\alpha_{1}, \alpha_{2}, \ldots, \alpha_{P}$ and $\beta=\beta_{1}, \beta_{2}, \cdots, \beta_{P}$ ( $P$ roots on the unit circle).The information of azimuth is included in $\alpha$ and $\beta$. Also, $\beta$ includes the information of azimuth and range. To obtain the correct bearing and range estimation, the parameter pairing is needed, which is investigated in [16]. Then, we obtain the bearing and range by

$$
\begin{aligned}
& \theta_{p}=\sin ^{-1}\left(\operatorname{angle}\left(\alpha_{p}\right) \lambda_{p} /(4 \pi d)\right) \\
& r_{p}=2 \pi d^{2} \cos ^{2} \theta_{p} /\left(\lambda \operatorname{angle}\left(\beta_{p}\right)\right)
\end{aligned}
$$

for $p=1, \cdots, P$.

\section{Simulation Results}

We assume a uniform linear array of 10 sensors with the spacing of array element is $1 / 4$ of wavelength, the number of snapshots $N=256$, the window length $h=129$. The starting and ending frequencies of signal 1 are 0.1 and 0.5 , and the two frequencies of signal 2 are 0 and 0.4 , respectively. The two LFM signals are $s_{1}(t)=e^{j 2 \pi\left(0.1 t+0.2 t^{2} / N\right)}$ and $s_{2}(t)=e^{j 0.4 \pi t^{2} / N}$.
The PWVD of the two signals are demonstrated in Fig.3.

Experiment 1: Suppose the two signals are positioned at $\theta_{1}=20^{\circ}, r_{1}=0.2 \lambda$ and $\theta_{2}=40^{\circ}, r_{2}=0.4 \lambda$, respectively. 100 Monte Carlo simulations are performed. Fig.4. shows the RMSE of bearing estimation of signal 1 and signal 2, respectively, using the proposed method and the method in [15]. SNR ranges from $0 \mathrm{~dB}$ to $20 \mathrm{~dB}$. Fig.5. shows the RMSE of range estimation.

From the figures, we conclude that when the signals are LFM signals, the method proposed in this paper has higher estimation accuracy than the method in [15].

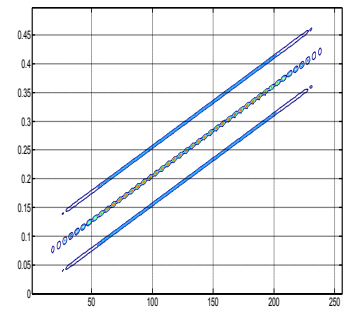

(a) $\mathrm{SNR}=10 \mathrm{~dB}$

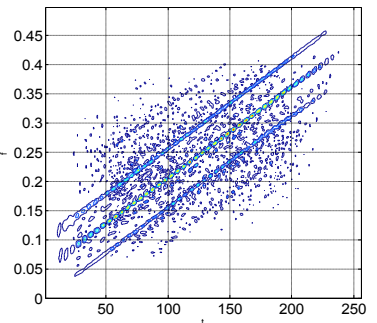

(b) $\mathrm{SNR}=0 \mathrm{~dB}$
Fig. 3. PWVD of two LFM signals

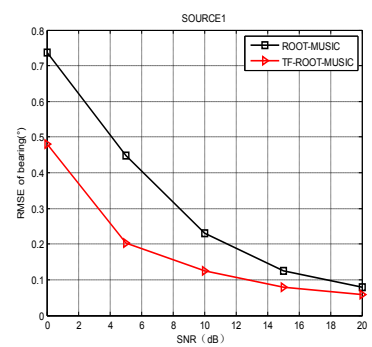

(a) source 1

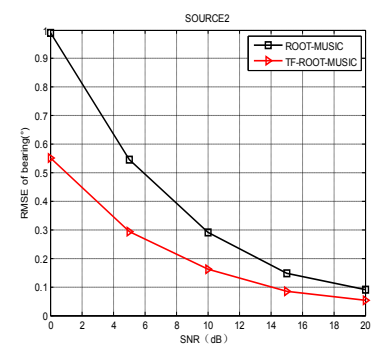

(b) source 2
Fig. 4. RMSE of bearing

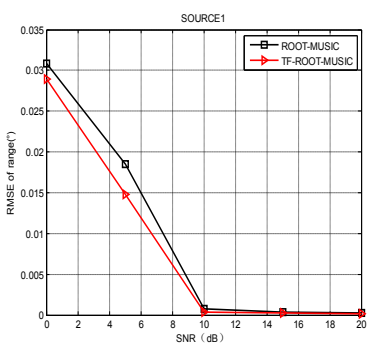

(a) source 1

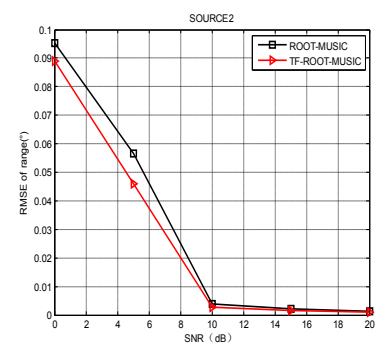

(b) source 2
Fig.5. RMSE of range

Experiment 2: In the example, we compare the bearing resolution of the proposed method and the method in [15]. Assume the bearing spacing between the two signals varies from $0^{\circ}$ to $10^{\circ}$ with $2^{\circ}$ difference, i.e., $\theta_{1}=20^{\circ}$, while $\theta_{2}$ is $20^{\circ}, 22^{\circ}, 24^{\circ}, 26^{\circ}, 28^{\circ}$ and $30^{\circ}$, respectively. $\mathrm{SNR}=20 \mathrm{~dB}$. We observe the performance of the RMSE of bearing and range estimation versus the bearing spacing between the two signals. The results are shown in Figs. 6 and 7, respectively.

It is shown that when the bearings are closely spaced, the proposed method has higher estimation resolution. 


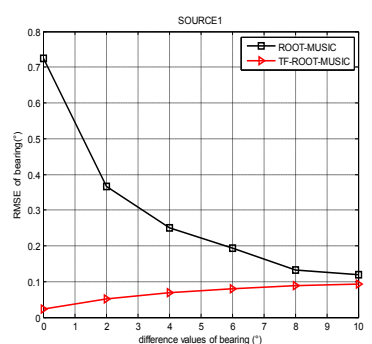

(a) source 1

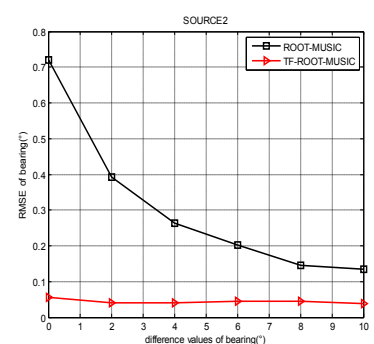

(b) source 2
Fig. 6. RMSE of bearing versus bearing spacing between the two signals

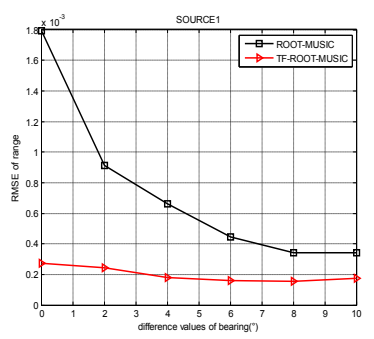

(a) source 1

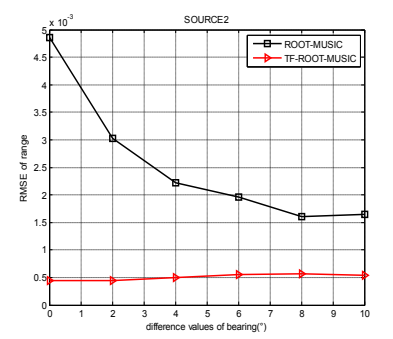

(b) source 2
Fig.7. RMSE of range versus bearing spacing between the two signals

Experiment 3: In the example, we similarly compare the range resolution of the proposed method and the method in [15]. Assume the range spacing between the two signals varies from $0 \lambda$ to $1.0 \lambda$ with $0.2 \lambda$ difference, i.e., $r_{1}=0.2 \lambda$, while $r_{2}$ is $0.2 \lambda, 0.4 \lambda, 0.6 \lambda, 0.8 \lambda, 1.0 \lambda, 1.2 \lambda$, respectively. $\mathrm{SNR}=20 \mathrm{~dB}$. We observe the RMSE of bearing and range estimation versus the range spacing between the two signals. The results are shown in Figs. 8 and 9, respectively.

It reveals that the proposed method has higher resolution in both bearing and range estimation when the two signals have close ranges.

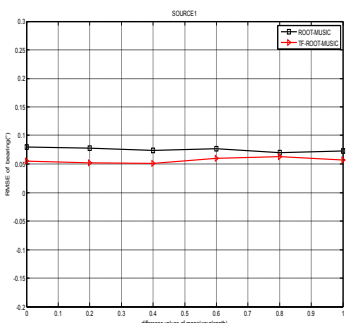

(a) source 1

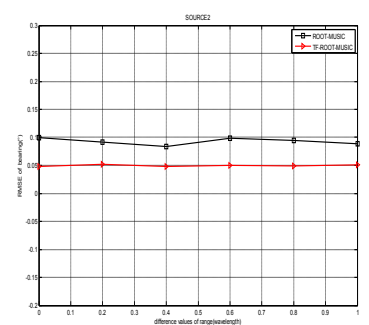

(b) source 2
Fig. 8. RMSE of bearing versus range spacing between the two signals

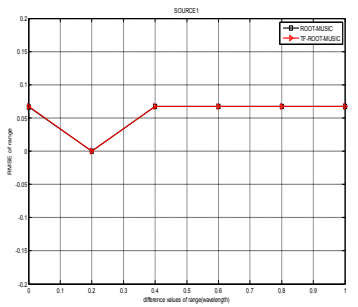

(a) source 1

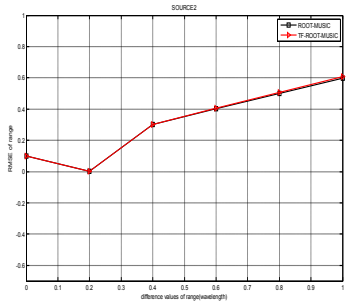

(b) source 2
Fig. 9. RMSE of range versus range spacing between the two signals

\section{Conclusion}

In this paper, a novel time-frequency Root-MUSIC method for near-field source localization is proposed. The simulations show that, for the bearing and range estimation of near-field non-stationary signals, the time-frequency based method has higher estimation accuracy and resolution than the conventional second order statistics based Root-MUSIC method, especially when the SNR is low, or the bearings or ranges of the sources are closely spaced.

\section{Acknowledgment}

The research is supported by Chinese National Natural Science Fund (61071140 and 60901060).

\section{References}

[1] Rodney A, Darren B, Thushara D. "Nearfield beamforming using radial reciprocity [J]". IEEE Trans. Signal Processing, 47(1), 33-40, January 1999.

[2] Krim H, viberg M. "Two decades of array processing research: The parametric approach [J]." IEEE Signal Processing Magazine, 13(4), July 1996.

[3] Lee J H, Lee C M, Lee K K. "A modified path-following algorithm using a known algebraic path [J]". IEEE Trans. Signal Processing, 47(5), May 1999.

[4] Yuntao W, Yi D, Guisheng L. "Jointly estimating both range and DOA of near field source [J]". Journal of Electronics (in Chinese). Mar. 2004

[5] Ju-Hong Lee, C.-H. Tung. "Estimating the bearings of near-field cyclostationary signals [J]". IEEE Trans.Signal Processing, 50(1), 110118, January 2002.

[6] Yang, Y., Peng, Z.K., Meng, G., Zhang, W.M., "Spline-Kernelled Chirplet Transform for the Analysis of Signals With Time-Varying Frequency and Its Application", IEEE Trans. Industrial Electronics, 59 (3), 1612-1621, 2012.

[7] Marelli, D., Aramaki, M., Kronland-Martinet, R., Verron, C., "An Efficient Time-Frequency Method for Synthesizing Noisy Sounds With Short Transients and Narrow Spectral Components", IEEE Transactions on Audio, Speech, and Language Processing, 20(4), 1400-1408, 2012.

[8] Zhang, J.J., Papandreou-Suppappola, A., Gottin, B., Ioana, C. "TimeFrequency Characterization and Receiver Waveform Design for Shallow Water Environments", IEEE Trans. Signal Processing, 57 (8), 29732985, 2009.

[9] Moeness G. Amin "Spatial time-frequency distributions for direction finding and blind source separation", Proc.SPIE, 62-73, 1999.

[10] Yimin Zhang, Weifeng Wu, Moeness G.Amin, "Subspace analysis of spatial time-frequency distribution matrices[J]", IEEE Trans. Signal Processing, 49(4), April 2001.

[11] Moeness G.Amin, Yimin Zhang, "Direction finding based on spatial time-frequency distribution matrices[J]", Signal Processing, 10(4), 325$339,2000$.

[12] L.A.Cirillo,A.M.Zoubir,and M.G.Amin, "Estimation of near-field parameters using spatial time-frequency distributions[C] ", IEEE Int.Conf.Acoust. Speech Signal Processing, 1141-1144, April 2007.

[13] B. Dominique and R. Cedric, "Time-frequency analysis of near-field optical data for extracting local attributes", IEEE Int.Conf.Acoust. Speech Signal Processing, 3565-3568, 2001.

[14] Karim Abed-Meraim,Yingbo Hua,Adel Belouchrani, "Second-order near-field source localization: algorithm and performance analysis[C]", Proceedings of Asilomar Conference, Pacific Grove, CA,USA, 723-727, 1996.

[15] Xuezhi Yan, "The Near Field Wireless Localization Algorithms and Key Technology of AGV Based on Ultrasonic Localization", Jilin University, $\mathrm{PhD}$ thesis, Chapter IV, 32-36, 2008.

[16] Grosicki E,Abed-Meraim K,A. "A weighted linear prediciton method for near-field source localization[C].” ICASSP, 2002, 3: 2957-2960. 